\title{
SOME UNIQUENESS THEOREMS FOR DIFFERENTIAL EQUATIONS WITH OPERATOR COEFFICIENTS
}

\author{
BY \\ ROBERT CARROLL AND JEROME NEUWIRTH
}

1. There has been in recent years a certain interest in differential equations with operator coefficients (see e.g. $[7 ; 10 ; 11 ; 16 ; 21 ; 12 ; 14 ; 25 ; 26 ; 28 ; 29 ; 31]$ ). In this paper we will present an adaptation of some methods of Calderón, Mizohata, and Malgrange for uniqueness in the Cauchy problem (see e.g. [8; 9 ; $17 ; 19])$ to the equation

$$
P u=\sum_{i=0}^{m} A_{i} u^{(i)}=0, u^{(i)}(0)=0(i=0, \cdots, m-1),
$$

where $u^{(i)}=d^{i} u / d t^{i}, A_{m}=I, A_{0}=P_{0}(\Lambda)\left(P_{0}\right.$ a polynomial of degree $\left.m\right)$ and $\Lambda, A_{i}(i=1, \cdots, m-1)$ are unbounded operators in a Hilbert space $H$ (precise hypotheses are given below). It is assumed that $t \rightarrow u(t) \in \mathscr{E}^{m}(H)$ with $t \rightarrow A_{i} u^{(i)} \in \mathscr{E}^{0}(H)\left(\mathscr{E}^{k}(H)\right.$ is the space of $k$-times continuously differentiable functions with values in $H)$, and that $\Lambda^{-1}=\Sigma \in \mathscr{L}(H)(\mathscr{L}(H)$ is the space of bounded linear operators in $H$ ). Suppose for example that $\Sigma^{m-i} A_{i} \subset A_{i} \Sigma^{m-i}$ with $\tilde{A}_{i}=A_{i} \Lambda^{-(m-i)}$ densely defined on $D\left(A_{i}\right)$ and bounded there; then $\tilde{A}_{i}$ determines an operator in $\mathscr{L}(H)$ by extension to $H$ which we denote again by $\tilde{A}_{i}$. We will regard $\Lambda$ as the basic operator and restrict the behavior of the $A_{i}$ relative to $\Lambda ; \Lambda^{m}$ is presumed to be densely defined and we note that $\Lambda^{-1} \in \mathscr{L}(H)$ implies $\Lambda$ is closed since $u_{n} \rightarrow u$ and $\Lambda u_{n} \rightarrow v$ imply $u=\Lambda^{-1} v$. It is seen also that $\Lambda^{k}$, $k \leqq m$, is now densely defined and closed. In practice the basic operator will be $\Lambda^{m}$ and if $\Lambda^{m}=A$ is closed with $\|\lambda R(\lambda,-A)\|<M<\infty$ then a closed $A^{1 / m}$ can be defined (see [5]).

Suppose now only that $u^{(i)} \in D\left(\Lambda^{m-t}\right)$ and set $u_{i+1}=\Lambda^{m-i-1} u^{(i)}(i=0, \cdots, m-1)$. Let $\tilde{A}_{i}$ be bounded operators (which may or may not arise from the preceding considerations) and assume $\tilde{A}_{i} \Lambda \subset \Lambda \tilde{A}_{i}$ with all $\tilde{A}_{i}$ and $\Sigma$ commuting. (These hypotheses will hold throughout the paper along with $\Lambda^{-1} \in \mathscr{L}(H)$ and $D\left(\Lambda^{m}\right)$ dense.) Then (1.1) leads us to pose the following problem for $t \rightarrow u(t) \in \mathscr{E}^{m}(H)$, $t \rightarrow \Lambda^{m-i} u^{(i)} \in \mathscr{E}^{0}(H)$,

$$
u^{(m)}+\Lambda \sum_{i=0}^{m-1} \tilde{A}_{i} u_{i+1}=0
$$

Presented to the Society, September 25, 1962; received by the editors January 9, 1963. 
This may be written in the form

$$
\begin{gathered}
d \mathbf{u} / d t+\Lambda H \mathbf{u}=0, \mathbf{u}=\left[\begin{array}{c}
u_{1} \\
\vdots \\
u_{m}
\end{array}\right], \mathbf{u}(0)=0, \\
H=\left[\begin{array}{cccc}
0 & -1 & \cdots & 0 \\
& & \cdots & \\
0 & 0 & \cdots & -1 \\
\tilde{A}_{0} & \tilde{A}_{1} & \cdots & \tilde{A}_{m-1}
\end{array}\right] .
\end{gathered}
$$

In order to pass strictly from (1.1) to (1.3) it is necessary that say $D\left(A_{i}\right) \subset D\left(\Lambda^{m-i}\right)$ and that the remaining hypotheses of paragraph one are fulfilled. In such a case uniqueness in the problem (1.3) implies uniqueness in the problem (1.1).

The characteristic polynomial of $H$ is formally

$$
|\lambda I-H|=(-1)^{m} \sum_{i=0}^{m}(-\lambda)^{i} \tilde{A}_{i}=(-1)^{m} q(\lambda)
$$

and we write $\tilde{A}_{0}\left(=A_{0} \Lambda^{-m}\right)=Q_{0}(\Lambda)=q_{0}(\Sigma)$ where the degree of $q_{0}$ may be any integer $r, 0 \leqq r \leqq m\left(q_{0}\right.$ has nonzero constant term in any case). The nature of the roots $\lambda_{i}$ of $q(\lambda)=0$ will play a crucial role in the theory (cf. $[8 ; 17 ; 19]$ ). Some of the present results were summarized in [20].

2. Let $\mathscr{B}$ be the commutative Banach algebra generated by the $m+1$ elements $a_{i}, a_{0}=\Sigma, a_{i}=\tilde{A_{i}}(i=1, \cdots, m-1), a_{m}=I$. The determination of characteristic roots of $q(\lambda)$ thus involves constructing algebraic functions of several Banach algebra elements. To do this we follow $[2 ; 3 ; 4 ; 22 ; 24 ; 30]$. The joint spectrum $\sigma(a, \mathscr{B}), a=\left(a_{0}, \cdots, a_{m-1}\right)$, of the elements $a_{i}(0 \leqq i \leqq m-1)$ is defined to be the set of all $\lambda \in \mathbb{C}^{m}$ for which there do not exist $b_{i} \in \mathscr{B}$ with $\Sigma_{0}^{m-1}\left(a_{i}-\lambda_{i}\right) b_{i}=1$ (see e.g. $[2 ; 3]$ ). Let $\hat{a}_{i}$ be the functions on the carrier space $\Phi_{\mathscr{B}}$ (see [22]) defined by the $a_{i}$, i.e., $\phi\left(a_{i}\right)=\hat{a}_{i}(\phi)$, and let $\alpha: \Phi_{\mathscr{B}} \rightarrow \mathbb{C}^{m}$ be defined by $\alpha(\phi)=\left(\hat{a}_{0}(\phi), \cdots, \hat{a}_{m-1}(\phi)\right)$. Then the range of $\alpha$ coincides with $\sigma(a, \mathscr{B})$ (see $[2 ; 3 ; 22])$. Since the $a_{i}(0 \leqq i \leqq m-1)$ with $a_{m}=I$ generate $\mathscr{B}, \alpha$ is a homeomorphism (see [22, pp. 113-114]); moreover $\sigma(a, \mathscr{B})$ is compact and is contained in the compact polydisc $\Delta=\prod_{0}^{m-1} D_{i}, D_{i}=\left\{z_{i}:\left|z_{i}\right| \leqq v\left(a_{i}\right)\right\}(i=0, \cdots, m-1)$, where $v\left(a_{i}\right)$ is the spectral radius $v\left(a_{i}\right)=\lim \left\|a_{i}\right\|^{1 / n}=\max \left|z_{i}\right|, z_{i} \in \sigma\left(a_{i}, \mathscr{B}\right)$ (see [22]). Since $v\left(a_{i}\right) \leqq\left\|a_{i}\right\|, \sigma(a, \mathscr{B})$ is surely contained in any open polydisc of the form $U^{\varepsilon}=\prod_{0}^{m-1} D_{i}^{\varepsilon}$ where $D_{i}^{\varepsilon}=\left\{z_{i}:\left|z_{i}\right|<\left\|a_{i}\right\|+\varepsilon\right\}$.

Let now $\mathscr{H}(U, \mathscr{B})$ be the class of holomorphic functions on $U^{\mathfrak{\varepsilon}}$ with valuesin $\mathscr{B}$. Then there is a continuous algebra homomorphism $J: \mathscr{H}\left(U^{\varepsilon}, \mathscr{B}\right) \rightarrow \mathscr{B}$ such that $J(b)=b$ for each "constant" $b \in \mathscr{B}$ and $J\left(z_{i}\right)=a_{i}$ where $z_{i}$ is the $i$ th coordinate function in $\mathbb{C}^{m}$ (see $\left.[2 ; 3]\right)$. This induces a homomorphism $J: \mathscr{H}\left(U^{\varepsilon}, \mathbb{C}\right) \rightarrow \mathscr{B}$ and we may write $\hat{J}(f)=f\left(a_{0}, \cdots, a_{m-1}\right)$; in fact $\hat{J}(f)$ may be represented by a multiple Cauchy integral over the distinguished boundary of say $\bar{U}^{\varepsilon / 2}=\prod_{0}^{m-1} \bar{D}_{i}^{\varepsilon / 2}$ 
(see $[2 ; 3 ; 22 ; 30]$ ). The homomorphism $\hat{J}$ is clearly characterized by the properties $\hat{J}(1)=1, \hat{J}\left(z_{i}\right)=a_{i}$, since $f$ may be approximated by polynomials uniformly on $\bar{U}^{\varepsilon / 2}$ (see e.g. [6]; cf. [2;3;30]).

REMARK 1 . The proof in [22] used a hypothesis of semi-simplicity for $\mathscr{B}$ in order to establish the homomorphism $\hat{J}$ and this hypothesis was therefore used in an earlier version of this paper. However as was shown in [2] such an assumption is unnecessary (cf. also [30]).

Thus we are led to consider the equation (see (1.5))

$$
q(\lambda, z)=q_{0}\left(z_{0}\right)+\sum_{i=1}^{m-1}(-\lambda)^{i} z_{i}+(-\lambda)^{m}=0
$$

in the $m$ complex variables $z_{i}(i=0, \cdots, m-1)$. If we can solve (2.1) for functions $\lambda_{j}(z)$ analytic in say $U^{\varepsilon}$ then we can determine operators $\lambda_{j}(a)=\hat{J}\left(\lambda_{j}(z)\right) \in \mathscr{B}$ such that $\hat{J}\left(q\left(\lambda_{j}(z), z\right)\right)=q\left(\lambda_{j}(a)\right)=0$. (We will not consider in this paper the possibility of roots $\lambda_{j} \in \mathscr{L}(H)$ of $q(\lambda)=0$ arising which are not given in terms of analytic functions on some $U^{\varepsilon}$.) Suppose that $q(\lambda)=(-1)^{m} \Pi\left(\lambda-\lambda_{i}\right)$ where each factor $\left(\lambda-\lambda_{i}\right)$ occurs $m_{i}$ times and suppose that this corresponds to a factorization $q(\lambda, z)=(-1)^{m} \prod\left(\lambda-\lambda_{i}(z)\right), \quad \lambda_{i}(z) \in \mathscr{H}\left(U^{\varepsilon}, \mathbb{C}\right)$. Writing formally $P u=\left(\Sigma_{0}^{m} A_{i}(d / d t)^{i}\right) u\left(A_{m}=I\right)$ it is seen that formally

$$
P u=\left(\sum A_{i} \Lambda^{-(m-i)}\left(\Lambda^{-1} d / d t\right)^{i} \Lambda^{m}\right) u=\left(\sum \tilde{A}_{i}\left(\Lambda^{-1} d / d t\right)^{i}\right) \Lambda^{m} u
$$

and therefore since $q(\lambda)=\Sigma(-\lambda)^{i} \tilde{A}_{i}$

$$
P u=(-1)^{m} \prod\left(-\Lambda^{-1} d / d t-\lambda_{i}\right) \Lambda^{m} u=\prod\left(d / d t+\lambda_{i} \Lambda\right) u .
$$

Without regard for the formal steps leading to (2.2) it is easily seen now that the last term in (2.2) is a valid decomposition (note $\lambda_{i} \in \mathscr{B}$ and it will be shown below that $\mathscr{B} \Lambda \subset \Lambda \mathscr{B})$. Indeed if $u \in \mathscr{E}^{1}(H)$ then $\left(\lambda_{i} u\right)^{\prime}=\lambda_{i} u^{\prime}$ trivially. Moreover if $\omega^{\prime} \in D(\Lambda), \omega(0)=0$, then (see [28]) $\Lambda \omega(t)=\Lambda \int_{0}^{t} \omega^{\prime} d \xi=\int_{0}^{t} \Lambda \omega^{\prime} d \xi$; therefore $(\Lambda \omega)^{\prime}=\Lambda \omega^{\prime}$. Hence $\prod\left(d / d t+\lambda_{i} \Lambda\right) u$ may be expanded to give $P u$. We remark that this factorization will be used in connection with the problem (1.3) and thus no assumption $u^{(i)} \in D\left(A_{i}\right)$ is made in general; by $P u$ in the following we will understand the form (2.2) (last term) with $u^{(i)} \in D\left(\Lambda^{m-i}\right)$. It remains to prove $\mathscr{B} \Lambda \subset \Lambda \mathscr{B}$. By assumption $a_{i} \Lambda \subset \Lambda a_{i}(i=0, \cdots, m-1)$ and hence for any polynomial $p\left(a_{i}\right) \in \mathscr{B}, p \Lambda \subset \Lambda p$. By definition $\mathscr{B}$ is the norm closure of polynomials in the $a_{i}$ and $I$; let $b \in \mathscr{B}$ and $p_{n} \rightarrow b$ in norm. If $u \in D(\Lambda)$ then

$$
b \Lambda u-\Lambda p_{n} u=\left(b-p_{n}\right) \Lambda u \rightarrow 0 ;
$$

therefore $p_{n} u \rightarrow b u$ and $\Lambda p_{n} u \rightarrow b \Lambda u$. This implies that $b u \in D(\Lambda)$ and $\Lambda b u=b \Lambda u$ since $\Lambda$ is closed; hence $\mathscr{B} \Lambda \subset \Lambda \mathscr{B}$. We will use the factorization (2.2) in $\S 4$ which is modeled after [19].

REMARK 2 . We note that in case $A_{i}=P_{i}(\Lambda)$ where $P_{i}$ is a polynomial of degree $m-i$ the theory takes on a particularly simple and interesting form. In this case 
$\tilde{A}_{i}=A_{i} \Lambda^{-(m-i)}=Q_{i}(\Lambda)=q_{i}(\Sigma)$ is a polynomial of degree $m-i$ in $\Sigma$. Thus $q(\lambda)=\Sigma(-\lambda)^{i} q_{i}(\Sigma)\left(q_{m}=1\right), q(\lambda, z)=\Sigma(-\lambda)^{i} q_{i}(z)$, and the $\lambda_{i}(z)$ are algebraic functions of one complex variable $z$. The Puiseux series (see [1]) for $\lambda_{i}$ are convergent and a detailed study of possible roots $\lambda_{i} \in \mathscr{B}$ (or $\lambda_{i} \in \mathscr{L}(H)$ ) can be envisioned using the many classical results available in this situation. Some examples will be given later.

In the following a separate treatment will be given for the case of distinct roots $\lambda_{i}$ because of its traditional importance. We note that distinct analytic functions $\lambda_{i}(z)$ and $\lambda_{j}(z)$ on $U^{\varepsilon}$ do not necessarily give rise to distinct operators $\lambda_{i}(a)$ and $\lambda_{j}(a)$. Suppose however that $\lambda_{i}(z) \neq \lambda_{j}(z)$ anywhere on $U^{\varepsilon}$; then $\left[\lambda_{i}(z)-\lambda_{j}(z)\right]^{-1}$ is analytic on say $U^{z / 2}$ and determines an operator $\left[\lambda_{i}(a)-\lambda_{j}(a)\right]^{-1}$. This means in particular that $\lambda_{i}(a) \neq \lambda_{j}(a)$. A criterion for distinct factors of this type can be given as follows. We recall that the discriminant $D(z)$ of $q(\lambda, z)$ is defined as $D(z)=\prod_{i<j}\left(\lambda_{i}(z)-\lambda_{j}(z)\right)^{2}$; moreover if $R\left(q, q^{\prime}\right)$ is the resultant of $q$ and $q^{\prime}$ then $R\left(q, q^{\prime}\right)=(-1)^{m} D(z)$ (see [27]; $q^{\prime}=d q / d \lambda, z$ is fixed). Therefore $D(z)$ is a polynomial in the $z_{i}$ (recall $q_{0}\left(z_{0}\right)$ is a polynomial) and its zeros determine an algebraic set in $\mathbb{C}^{m}$. If all the zeros of $D(z)$ lie outside of $U^{\varepsilon}$ then for any $z \in U^{\varepsilon}$ fixed there will be $m$ distinct analytic roots $\lambda_{i}(z)$ of $q(\lambda, z)=0$ in some neighborhood of $z$ (see [13] for the implicit function theorem; cf. also [6]). We can now determine by analytic continuation $m$ distinct analytic functions $\lambda_{i}(z)$ on say $U^{2 \varepsilon / 3}$ with $\lambda_{i}(z) \neq \lambda_{j}(z)$ anywhere (see e.g. $\left.[6 ; 13]\right)$. This proves

Proposition 1. Assume all zeros of $D(z)$ lie outside $U^{z}$.Then there exist $m$ distinct (single-valued) analytic functions $\lambda_{i}(z)$ in say $U^{2 \varepsilon / 3}$, roots of $q(\lambda, z)=0$, such that $\lambda_{i}(z) \neq \lambda_{j}(z)$ anywhere in $U^{2 z / 3}$. These functions determine $m$ distinct operators $\lambda_{i}(a) \in \mathscr{B}$ with $\left[\lambda_{i}(a)-\lambda_{j}(a)\right]^{-1} \in \mathscr{B}$ well defined.

Remark 2a (Added in proof). Some general existence results involving a spectral theory on $\sigma$ are indicated in [12a].

3. The case of distinct roots $\lambda_{i}(a)$ of the type described in Proposition 1 will be treated first. The presentation will follow that of $[8 ; 17]$. Let

$$
N^{-1}=\left[\begin{array}{cccc}
1 & 1 & \cdots & 1 \\
\left(-\lambda_{1}\right) & \left(-\lambda_{2}\right) & & \left(-\lambda_{m}\right) \\
& & \cdots & \\
\left(-\lambda_{1}\right)^{m-1} & \left(-\lambda_{2}\right)^{m-1} & \cdots & \left(-\lambda_{m}\right)^{m-1}
\end{array}\right] .
$$

Since $\left(\lambda_{i}-\lambda_{j}\right)^{-1} \in \mathscr{B}$ by assumption, the matrix $N$ such that $N N^{-1}=I$ may be computed (see [15]) and is seen to be a matrix of bounded operators in $\mathscr{B}$. Then

$$
\mathscr{H}=N H N^{-1}=\left(\left(\lambda_{i}\right)\right)
$$

is a diagonal matrix (see $[8 ; 15 ; 17])$. Setting $\mathbf{y}=N \mathbf{u}(1.3)$ becomes 


$$
d \mathbf{y} / d t+\mathscr{H} \Lambda \mathbf{y}=0, \mathbf{y}(0)=0
$$

(recall $\mathscr{B} \Lambda \subset \Lambda \mathscr{B}$ and thus $\mathscr{H} \Lambda \subset \Lambda \mathscr{H}$ ).

Suppose now that $\mathbf{v}$ satisfies $\mathbf{v}(0)=\mathbf{v}(\tau)=0$ and set $\omega=\exp (k / 2)(t-\tau)^{2} \mathbf{v}$; then $\omega(0)=\omega(\tau)=0$ and writing $\omega=\psi \mathbf{v}$,

$$
\omega_{t}-k(t-\tau) \omega+\mathscr{H} \Lambda \omega=\psi\left(\mathbf{v}_{t}+\mathscr{H} \Lambda \mathbf{v}\right) \text {. }
$$

(It will be neater to work with $\mathscr{H} \Lambda$ rather than $\Lambda \mathscr{H}$ as will be seen.) We write $\mathscr{H}=\mathscr{H}_{1}+\mathscr{H}_{2}, \quad \mathscr{H}_{1}=\frac{1}{2}\left(\mathscr{H}+\mathscr{H}^{*}\right), \quad \mathscr{H}_{2}=\frac{1}{2}\left(\mathscr{H}-\mathscr{H}^{*}\right), \quad$ and define the integral $I=\int_{0}^{\tau} \exp k(t-\tau)^{2}\left\|\mathbf{v}_{t}+\mathscr{H} \Lambda \mathbf{v}\right\|^{2} d t$. Then

$$
I=\int\left\|\omega_{t}+\mathscr{H}_{2} \Lambda \omega\right\|^{2} d t+\int\left\|\mathscr{H}_{1} \Lambda \omega-k(t-\tau) \omega\right\|^{2} d t
$$

$$
+k \int\|\omega\|^{2} d t+J_{1}+J_{2}
$$

$$
\begin{aligned}
& J_{1}=-2 \operatorname{Re} \int k(t-\tau)\left(\mathscr{H}_{2} \Lambda \omega, \omega\right) d t \\
& J_{2}=2 \operatorname{Re} \int\left(\omega_{t}+\mathscr{H}_{2} \Lambda \omega, \mathscr{H}_{1} \Lambda \omega\right) d t
\end{aligned}
$$

(all integrals are over $[0, \tau])$. We note that $\mathscr{H}_{1}=\mathscr{H}_{1}^{*}, \mathscr{H}_{2}^{*}=-\mathscr{H}_{2}$, and

$$
-2 \operatorname{Re} \int k(t-\tau)\left(\omega_{t}, \omega\right) d t=k \int\|\omega\|^{2} d t .
$$

It will be assumed now that $D(\Lambda) \subset D\left(\Lambda^{*}\right)$ and that $\Lambda^{*}-\Lambda$ is bounded on $D(\Lambda)$. Then $\Lambda^{*}-\Lambda$ may be extended to a bounded operator on $H$ denoted by the same symbol. Since $\mathscr{B} \Lambda \subset \Lambda \mathscr{B}$ it follows that $\mathscr{B}^{*} \Lambda^{*} \subset \Lambda^{*} \mathscr{B} *$ (see e.g. [23]); therefore $\Lambda^{*} \mathscr{H}^{*} \omega$ is well defined. Similarly $\Lambda^{*} \mathscr{H} \omega=\left[\left(\Lambda^{*}-\Lambda\right)+\Lambda\right] \mathscr{H} \omega$ is defined since $\omega \in D(\Lambda)$. We define now the operator on $D(\Lambda)$

$$
T_{1}=\Lambda^{*} \mathscr{H}_{2}-\mathscr{H}_{2} \Lambda=\frac{1}{2}\left\{\left(\Lambda^{*}-\Lambda\right) \mathscr{H}+\mathscr{H}^{*}\left(\Lambda-\Lambda^{*}\right)\right\}
$$

and extend it to $H$ by continuity (note $\mathscr{H} \Lambda \subset \Lambda \mathscr{H}$ and $\Lambda^{*} \mathscr{H}^{*}=\mathscr{H}^{*} \Lambda^{*}$ on $D(\Lambda))$. Then

$$
\begin{aligned}
& J_{1}=k \int(t-\tau)\left(\omega, T_{1} \omega\right) d t, \\
&\left|J_{1}\right| \leqq k \int(\tau-t)\|\omega\|\left\|T_{1} \omega\right\| d t \leqq k \tau c_{1} \int\|\omega\|^{2} d t .
\end{aligned}
$$

Turning now to $J_{2}$ we note first (see [8]) 


$$
\frac{d}{d t}\left(\mathscr{H}_{1} \Lambda \omega, \omega\right)=\lim _{h \rightarrow 0}\left\{\left(\frac{\omega(t+h)-\omega(t)}{h}, \Lambda^{*} \mathscr{H}_{1}^{*} \omega(t+h)\right)\right.
$$

$$
\left.+\left(\Lambda \omega(t), \mathscr{H}_{1}^{*}\left(\frac{\omega(t+h)-\omega(t)}{h}\right)\right)\right\}
$$

$$
\frac{d}{d t}\left(\mathscr{H}_{1} \Lambda \omega, \omega\right)=\left(\omega_{t}, \Lambda^{*} \mathscr{H}_{1} \omega\right)+\left(\Lambda \omega, \mathscr{H}_{1} \omega_{t}\right)
$$

(note in general $\omega_{t} \notin D(\Lambda)$ ). From this it follows that

$$
2 \operatorname{Re} \int\left(\omega_{t}, \mathscr{H}_{1} \Lambda \omega\right) d t=\int\left(\omega_{t}, T_{2} \omega\right) d t
$$

defining $T_{2}=\mathscr{H}_{1} \Lambda-\Lambda^{*} \mathscr{H}_{1}$. We display $T_{2}$ as a bounded operator by writing

$$
T_{2}=\frac{1}{2}\left\{\mathscr{H}^{*}\left(\Lambda-\Lambda^{*}\right)-\left(\Lambda^{*}-\Lambda\right) \mathscr{H}\right\}
$$

Now $J_{2}$ may be written

$$
\begin{aligned}
J_{2} & =\int\left(\omega_{t}+\mathscr{H}_{2} \Lambda \omega, T_{2} \omega\right) d t+\int\left(\Lambda \omega, T_{3} \omega\right) d t \\
T_{3} & =\left(\mathscr{H}_{1} \mathscr{H}_{2}-\mathscr{H}_{2} \mathscr{H}_{1}\right) \Lambda+\mathscr{H}_{2} T_{2}=T_{4}+\mathscr{H}_{2} T_{2}, \\
T_{4} & =\frac{1}{2}\left(\mathscr{H}^{*} \mathscr{H}-\mathscr{H} \mathscr{H}^{*}\right) \Lambda .
\end{aligned}
$$

We want to find conditions under which $T_{4}$ (defined on $D(\Lambda)$ ) will be a bounded operator. For example if the $a_{i}$ are normal operators then $T_{4}=0$. First however we summarize the preceding.

Proposition 2. Assume $I$ defined as above with $\omega(0)=\omega(\tau)=0$, $D(\Lambda) \subset D\left(\Lambda^{*}\right)$, and $\Lambda^{*}-\Lambda$ bounded. Then $T_{1}$ and $T_{2}$ are bounded and the equations (3.10), (3.11), (3.16) hold.

Now as stated earlier the $\lambda_{i}(a)$ are given as the norm convergent Cauch y integrals of absolutely convergent multiple power series $\lambda_{i}(z), \lambda_{i}(z)$ analytic on say $U^{\varepsilon}$; hence the $\lambda_{i}(a)$ are represented by absolutely convergent power series in the $a_{i}$ (see $\left.[2 ; 6 ; 13 ; 22 ; 30]\right)$. Thus set $a=a_{0} \cdots a_{m-1}$ and $\lambda_{i}(a)=\sum c_{n}^{i} a^{n}$ $\left(n=\left(n_{0}, \cdots, n_{m-1}\right)\right)$; then $\lambda_{i}^{*}=\sum \bar{c}_{n}^{i} a^{* \bar{n}}\left(\bar{n}=\left(n_{m-1}, \cdots, n_{0}\right)\right)$ and

$$
T_{i}=\lambda_{i}^{*} \lambda_{i}-\lambda_{i} \lambda_{i}^{*}=\sum \bar{c}_{n}^{i} c_{k}^{i}\left(a^{* \bar{n}} a^{k}-a^{k} a^{* \bar{n}}\right)
$$

(see $[13$, p. 100]); the series (3.19) are absolutely convergent. The expressions 
$T_{i} \Lambda$ will determine the behavior of $T_{4}$; we observe that the constant term in $T_{i}$ is zero as well as any term involving one of the $a^{k}$ or $a^{* \bar{n}}$ a constant. Set now $G_{\bar{n}, k}=a^{* \bar{n}} a^{k}-a^{k} a^{* \bar{n}} ;$ then

$$
G_{n, k}=\sum_{l=0}^{m-1} a^{n^{\prime}}\left(a^{* n_{l}}-a^{n_{l}}\right) a^{* \bar{n}^{\prime \prime}} a^{k}+\sum_{l=0}^{m-1} a^{k} a^{* n^{\prime}}\left(a^{n_{l}}-a^{* n_{l}}\right) a^{\bar{n}^{\prime \prime}}
$$

where $\bar{n}^{\prime}=\left(n_{m-1}, \cdots, n_{l+1}\right), \bar{n}^{\prime \prime}=\left(n_{l-1}, \cdots, n_{0}\right)$. Note next that $\Lambda$ may be carried to the left in in $G_{\bar{n}, k} \Lambda$ as follows: $a^{* \bar{n}^{\prime \prime}} a^{k} \Lambda=a^{* \bar{n}^{\prime \prime}} \Lambda a^{k}=a^{* \bar{n}^{\prime \prime}}\left(\Lambda-\Lambda^{*}\right) a^{k}+\Lambda^{*} a^{* n^{\prime \prime}} a^{k}$. We note that in carrying $\Lambda$ to the left we start with $G_{\bar{n}, k} \Lambda$ defined on $D(\Lambda)$. Thus everything is well defined up to and including the term

$$
a^{* \bar{n}^{\prime \prime}} a^{k} \Lambda=a^{* \bar{n} "}\left(\Lambda-\Lambda^{*}\right) a^{k}+\Lambda^{*} a^{* \bar{n} "} a^{k}
$$

(recall $\mathscr{B} \Lambda \subset \Lambda \mathscr{B}$ and $\mathscr{B}^{*} \Lambda^{*} \subset \Lambda^{*} \mathscr{B}^{*}$ ). However we do not know whether $a^{* n^{\prime \prime}} a^{k} u \in D(\Lambda)$ for $u \in D(\Lambda)$ and thus a priori a further decomposition $\Lambda^{*} a^{* n "} a^{k}=\Lambda a^{* \bar{n}^{\prime \prime}} a^{k}+\left(\Lambda^{*}-\Lambda\right) a^{* \bar{n}^{\prime \prime}} a^{k}$ is meaningless. Suppose we only make assumptions which insure that $A \Lambda$ is bounded on $D(\Lambda)$ where $A=a^{* n_{l}}-a^{n_{l}}$; then $A \Lambda$ is extendable to $H$. Let $u \in D(\Lambda)$; then $a^{k} u \in D(\Lambda), v=a^{* \bar{n}^{\prime \prime}} a^{k} u \in D\left(\Lambda^{*}\right)$, and $A \Lambda^{*} v$ is defined. On $D(\Lambda)$ we have $A \Lambda^{*}=A \Lambda+A\left(\Lambda^{*}-\Lambda\right)=\Omega$ bounded and extendable to $H$. We want to check whether the extended $\Omega$ agrees with $A \Lambda^{*}$ on $D\left(\Lambda^{*}\right)$. Let $v \in D\left(\Lambda^{*}\right)$ and $u_{n} \rightarrow v, u_{n} \in D(\Lambda)$; then $\Omega u_{n}=A \Lambda^{*} u_{n} \rightarrow \omega$ by the continuity of $\Omega$. However there is no assurance that $\omega=A \Lambda^{*} v$ (unless say $A$ is invertible). Therefore we write

$$
\begin{gathered}
G_{\bar{n}, k} \Lambda=\sum_{i=0}^{m-1} a^{k} a^{* \bar{n}^{\prime}}\left(a^{n_{l}}-a^{* n_{l}}\right) \Lambda a^{\bar{n}^{\prime \prime}}+\sum_{l=0}^{m-1} a^{\bar{n}^{\prime}}\left(a^{* n_{l}}-a^{n_{l}}\right) \Lambda^{*} a^{* \bar{n}^{\prime \prime}} a^{k} \\
+\sum_{l=0}^{m-1} a^{\bar{n}^{\prime}}\left(a^{* n_{l}}-a^{n_{l}}\right) a^{* \bar{n}^{\prime \prime}}\left(\Lambda-\Lambda^{*}\right) a^{k}
\end{gathered}
$$

If $\left(a^{* n_{l}}-a^{n_{l}}\right) \Lambda$ is bounded on $D(\Lambda)$ and $\left(a^{* n_{l}}-a^{n_{l}}\right) \Lambda^{*}$ is bounded on $D\left(\Lambda^{*}\right)$, then the terms in the series for $T_{i} \Lambda$ are all bounded operators (extend $G_{\bar{n}, k} \Lambda$ to $H$ by continuity); however there is no a priori guarantee that the resulting series converges. If however $\Sigma \bar{c}_{n}^{i} c_{k}^{i}\left(G_{\bar{n}, k} \Lambda\right)=\Sigma\left(g_{\bar{n}, k}^{i} \Lambda\right)$ converges absolutely to an operator $S$, then for $u \in D(\Lambda)$

$$
S u=\lim \left(\Sigma g_{\bar{n}, k}^{i} \Lambda\right) u=\lim \left(\Sigma\left(g_{\bar{n}, k}^{i} \Lambda u\right)\right)=\lim \left(\sum g_{\bar{n}, k}^{i}\right) \Lambda u=T_{i} \Lambda u .
$$

Hence $S=T_{i} \Lambda$ on $D(\Lambda)$ and we define $T_{i} \Lambda$ on $H$ to equal $S$. Moreover since $\mathscr{L}(H)$ is a Banach space, if $\Sigma\left(g_{n} \frac{i}{n} \Lambda\right)$ converges absolutely, then there exists $S \in \mathscr{L}(H)$ to which it converges.

In order to find conditions for convergence we remark that

$$
\left(a_{i}^{* s}-a_{i}^{s}\right) \Lambda=\sum_{k=0}^{s-1} a_{i}^{* k}\left(a_{i}^{*}-a_{i}\right) \Lambda a_{i}^{s-k-1}
$$


and thus if $\left(a_{i}^{*}-a_{i}\right) \Lambda$ is bounded so is $\left(a_{i}^{* s}-a_{i}^{s}\right) \Lambda$. Moreover for $s$ large

$$
\begin{aligned}
\left\|\left(a_{i}^{* s}-a_{i}^{s}\right) \Lambda\right\| & \leqq s\left\|a_{i}\right\|^{s-1}\left\|\left(a_{i}^{*}-a_{i}\right) \Lambda\right\| \\
& \leqq(1+\delta)^{s}\left\|a_{i}\right\|^{s-1}\left\|\left(a_{i}^{*}-a_{i}\right) \Lambda\right\| .
\end{aligned}
$$

Therefore if $\left\|\left(a_{i}^{*}-a_{i}\right) \Lambda\right\| \leqq c\left\|a_{i}\right\|$, then

$$
\left\|\left(a_{i}^{* s}-a_{i}^{s}\right) \Lambda\right\| \leqq c\left[(1+\delta)\left\|a_{i}\right\|\right]^{s} .
$$

Similarly $\left(a_{i}^{* s}-a_{i}^{s}\right) \Lambda^{*}=-\left(a_{i}^{s}-a_{i}^{* s}\right) \Lambda^{*}=-\sum_{k=0}^{s-1} a_{i}^{k}\left(a_{i}-a_{i}^{*}\right) \Lambda^{*} a_{i}^{* s-k-1}$ and we want to make an assumption $\left\|\left(a_{i}^{*}-a_{i}\right) \Lambda^{*}\right\| \leqq c\left\|a_{i}\right\|$ on $D\left(\Lambda^{*}\right)$. We examine now the terms $G_{\bar{n}, k} \Lambda$ and see that the last term in (3.21) may be bounded by $2 c m \prod\left\|a_{i}\right\|^{n_{i}+k_{i}}$ and the first two by $c m \prod\left[(1+\delta)\left\|a_{i}\right\|\right]^{n_{i}+k_{i}}$ (c represents various constants at different times). Hence if $\varepsilon>\delta \max \left\|a_{i}\right\|$ and the $\lambda_{i}(z)$ are analy tic on $U^{e}$ it follows that $\Sigma\left(g_{\bar{n}, k}^{i} \Lambda\right)$ converges absolutely. This proves (since $\delta>0$ is arbitrary)

Proposition 3. Assume $\left\|\left(a_{i}^{*}-a_{i}\right) \Lambda\right\| \leqq c\left\|a_{i}\right\|$ on $D(\Lambda),\left\|\left(a_{i}^{*}-a_{i}\right) \Lambda^{*}\right\| \leqq c\left\|a_{i}\right\|$ on $D\left(\Lambda^{*}\right)$, and that the $\lambda_{i}(z)$, analytic on $U^{\varepsilon}$, define $\lambda_{i}(a)$. Then $T_{4}$ is a bounded operator.

COROLlaRY. The conclusion holds if only $\left\|\left(a_{i}^{*}-a_{i}\right) \Lambda^{*}\right\| \leqq c\left\|a_{i}\right\|$ on $D\left(\Lambda^{*}\right)$.

Proof. Let $u \in D(\Lambda)$ then

$$
\begin{aligned}
\left\|\left(a_{i}^{*}-a_{i}\right) \Lambda u\right\| & \leqq\left\|\left(a_{i}^{*}-a_{i}\right)\left(\Lambda-\Lambda^{*}\right) u\right\|+\left\|\left(a_{i}^{*}-a_{i}\right) \Lambda^{*} u\right\| \\
& \leqq 2 c_{1}\left\|a_{i}\right\|\|u\|+c\left\|a_{i}\right\|\|u\| .
\end{aligned}
$$

QED

REMARK 3. The estimates for the $T_{i}$ show that (and in fact are based upon the fact that) the individual terms in the $T_{i}$ are bounded, e.g., $T_{i} \Lambda$. This remark will be used later when the $\lambda_{i}$ are no longer assumed distinct.

From (3.16) the following estimates now hold if we assume $\mathscr{H}_{1}$ is invertible on its range.

$$
\begin{aligned}
\|\Lambda \omega\| & \leqq c_{2}\left\|\mathscr{H}_{1} \Lambda \omega\right\| \leqq c_{2}\left\{\left\|\mathscr{H}_{1} \Lambda \omega-k(t-\tau) \omega\right\|+\|k(t-\tau) \omega\|\right\}, \\
\left|J_{2}\right| & \leqq \frac{c}{2}\left\{\frac{1}{\sqrt{ } k} \int\left\|\omega_{1}+\mathscr{H}_{2} \Lambda \omega\right\|^{2} d t+\sqrt{ } k \int\|\omega\|^{2} d t\right\} \\
& +\frac{c_{2}}{2}\left\{\frac{1}{\sqrt{ } k} \int\left\|\mathscr{H}_{1} \Lambda \omega-k(t-\tau) \omega\right\|^{2} d t+\sqrt{ } k \int\|\omega\|^{2} d t+k \tau \int\|\omega\|^{2} d t\right\}
\end{aligned}
$$

(note $\int A B d t \leqq 1 / 2\left[\int A^{2} / \alpha^{2} d t+\int \alpha^{2} B^{2} d t\right]$ and set $\alpha=k^{1 / 4}$ ). Choosing $k$ large we make first $c / \sqrt{ } k \leqq 1$ and $c_{2} / \sqrt{ } k \leqq 1$; then from (3.5) (see also (3.11))

$$
\begin{aligned}
I \geqq & \frac{1}{2} \int\left\|\omega_{t}+\mathscr{H}_{2} \Lambda \omega\right\|^{2} d t+\frac{1}{2} \int\left\|\mathscr{H}_{1} \Lambda \omega-k(t-\tau) \omega\right\|^{2} d t \\
& +\left(k-\frac{k \tau c_{2}}{2}-\frac{c \sqrt{ } k}{2}-\frac{c_{2} \sqrt{ } k}{2}-k \tau c_{1}\right) \int\|\omega\|^{2} d t .
\end{aligned}
$$


Choose now $\tau$ small enough so that $\tau\left(c_{1}+c_{2} / 2\right)<1 / 3$; then make $k$ larger if necessary so that $\frac{1}{2}\left(c_{2}+c\right) \sqrt{ } k<k / 3$ (i.e., $\left.\sqrt{ } k>\frac{3}{2}\left(c+c_{2}\right)\right)$. Then

$$
\begin{aligned}
I \geqq \frac{1}{2} \int\left\|\omega_{t}+\mathscr{H}_{2} \Lambda \omega\right\|^{2} d t+\frac{1}{2} \int\left\|\mathscr{H}_{1} \Lambda \omega-k(t-\tau) \omega\right\|^{2} d t \\
\quad+\frac{k}{3} \int\|\omega\|^{2} d t .
\end{aligned}
$$

Next from (3.25) for $k_{1}<k, k_{1}$ fixed,

$$
\frac{k_{1}}{2 k} \int\left\|\mathscr{H}_{1} \Lambda \omega-k(t-\tau) \omega\right\|^{2} d t \geqq \frac{k_{1}}{4 k c_{2}^{2}} \int\|\Lambda \omega\|^{2} d t-\frac{k_{1} k \tau^{2}}{2} \int\|\omega\|^{2} d t
$$

If $\tau$ is now chosen smaller if necessary until $k_{1} \tau^{2} / 2<1 / 6$, then

$$
I \geqq \frac{\varepsilon}{k} \int\|\Lambda \omega\|^{2} d t+\frac{k}{6} \int\|\omega\|^{2} d t .
$$

The estimate (3.30) holds then for $k$ large ( $\tau$ may now be fixed). This proves

Proposition 4. Assume the hypotheses of Propositions 2 and 3 and suppose $\mathscr{H}_{1}$ is invertible. Then (3.30) holds.

If now $\mathbf{y}$ is a solution of the Cauchy problem (3.3); $d \mathbf{y} / d t+\mathscr{H} \Lambda \mathbf{y}=0, \mathbf{y}(0)=0$, let $\phi \in C^{\prime}[0, \infty), \phi=1$ for $t \leqq \tau / 2, \phi=0$ for $t \geqq \tau$, and set $\phi \mathbf{y}=\mathbf{v}$. Then $\mathbf{v}$ satisfies $\mathbf{v}(0)=\mathbf{v}(\tau)=0$ and

$$
\mathbf{v}_{t}+\mathscr{H} \Lambda \mathbf{v}=\phi_{t} \mathbf{y}
$$

Therefore defining $I$ as before

$$
I \leqq \int \exp k(t-\tau)^{2} \phi_{t}^{2}\|\mathbf{y}\|^{2} d t \leqq \hat{c} \int_{\tau / 2}^{\tau} \exp k(t-\tau)^{2} d t,
$$

where $\hat{c}$ depends on $\phi$ and $\mathbf{y}$. Using (3.30) we obtain in particular

$$
\frac{k}{6} \int_{0}^{\tau} \exp k(t-\tau)^{2}\|\mathbf{v}\|^{2} d t \leqq \hat{c} \int_{\tau / 2}^{\tau} \exp k(t-\tau)^{2} d t
$$

This can hold (for $k$ arbitrarily large) only if $\mathbf{v} \equiv 0$ on $[0, \tau / 2]$ (see $[17 ; 19]$, and note that the above development follows [17] quite closely). Therefore

THEOREM 1. Under the hypotheses of Proposition 4, if $\mathbf{y}$ is a solution of the Cauchy problem $\mathbf{y}_{t}+\mathscr{H} \Lambda \mathbf{y}=0, \mathbf{y}(0)=0$, then $\mathbf{y} \equiv 0$ in a neighborhood of $t=0$.

Suppose $\mathscr{H}_{1}=0$ (this will be called a hyperbolic case); then $J_{2}=0$ and we have the estimates

$$
I \geqq k\left(1-\tau c_{1}\right) \int\|\omega\|^{2} d t .
$$

Therefore it is easily seen that the following theorem holds, if $\mathscr{H}_{1}=0$. 
THEOREM 2. Under the hypotheses of Proposition 2, if $\mathbf{y}$ is a solution of $\mathbf{y}_{t}+\mathscr{H} \Lambda \mathbf{y}=0, \mathbf{y}(0)=0$, then $\mathbf{y} \equiv 0$ in a neighborhood of $t=0 \quad\left(\mathscr{H}_{1}=0\right)$.

4. In this section the factorization (2.2) will be used for the case of multiple roots following [19]. Assume $v^{(i)}(0)=v^{(i)}(\tau)=0 \quad(0 \leqq i \leqq m-1)$ and set $\omega=\exp (k / 2)(t-\tau)^{2} v$. Then writing again $\psi=\exp (k / 2)(t-\tau)^{2}$ consider a factor $\left(d / d t+\lambda_{i} \Lambda\right) v$.

$$
\omega_{t}-k(t-\tau) \omega+\lambda_{i} \Lambda \omega=\psi\left(v_{t}+\lambda_{i} \Lambda v\right) .
$$

We write $\lambda_{i}=\tilde{\lambda}_{i}+\hat{\lambda}_{i}$ with $\tilde{\lambda}_{i}=\frac{1}{2}\left(\lambda_{i}+\lambda_{i}^{*}\right), \quad \hat{\lambda}_{i}=\frac{1}{2}\left(\lambda_{i}-\lambda_{i}^{*}\right)$, and define $I_{j}=\int \psi^{2}\left\|v_{t}+\lambda_{j} \Lambda v\right\|^{2} d t$. Then

$$
\begin{aligned}
I_{j}= & \int\left\|\omega_{t}+\hat{\lambda}_{j} \Lambda \omega\right\|^{2} d t+\int\left\|\tilde{\lambda}_{j} \Lambda \omega-k(t-\tau) \omega\right\|^{2} d t \\
& +k \int\|\omega\|^{2} d t+J_{1}^{j}+J_{2}^{j}, \\
J_{1}^{j}= & -2 \operatorname{Re} \int k(t-\tau)\left(\hat{\lambda}_{j} \Lambda \omega, \omega\right) d t, \\
J_{2}^{j}= & 2 \operatorname{Re} \int\left(\omega_{t}+\hat{\lambda}_{j} \Lambda \omega_{1} \tilde{\lambda}_{j} \Lambda \omega\right) d t .
\end{aligned}
$$

It follows from the preceding analysis that under the hypotheses $D(\Lambda) \subset D\left(\Lambda^{*}\right)$, $\Lambda^{*}-\Lambda$ bounded, $\left\|\left(a_{i}^{*}-a_{i}\right) \Lambda^{*}\right\| \leqq c\left\|a_{i}\right\|, \lambda_{j}(z)$ analytic on $U^{\varepsilon}$, and $\tilde{\lambda}_{j}$ invertible, that (3.30) holds with $I$ replaced by $I_{j}$. (Since there are only a finite number of roots we may suppose that $\varepsilon, k, \tau$ in (3.30) do not depend on $j$.) Now we iterate this procedure, taking as a new $v$ the function $\hat{v}=v_{t}+\lambda_{j} \Lambda v$; then $\hat{v}(0)=\hat{v}(\tau)=0$, and setting $c=\min (\varepsilon, 1 / 6)$,

$$
\begin{aligned}
& \int \psi^{2}\left\|\left(\frac{d}{d t}+\lambda_{i} \Lambda\right)\left(\frac{d}{d t}+\lambda_{j} \Lambda\right) v\right\|^{2} d t \\
& \geqq c\left\{k \int \psi^{2}\left\|v_{t}+\lambda_{j} \Lambda v\right\|^{2} d t+\frac{1}{k} \int \psi^{2}\left\|\Lambda\left(v_{t}+\lambda_{j} \Lambda v\right)\right\|^{2} d t\right\} \\
& \geqq c^{2}\left\{k^{2} \int \psi^{2}\|v\|^{2} d t+2 \int \psi^{2}\|\Lambda v\|^{2} d t+\frac{1}{k^{2}} \int\left\|\Lambda^{2} v\right\|^{2} d t\right\} .
\end{aligned}
$$

Proposition 5. Assume $D(\Lambda) \subset D\left(\Lambda^{*}\right), \Lambda^{*}-\Lambda$ bounded, $\left\|\left(a_{i}^{*}-a_{i}\right) \Lambda^{*}\right\| \leqq$ $c\left\|a_{i}\right\|$ in $D\left(\Lambda^{*}\right), \lambda_{j}(z)$ analytic on $U^{\ell}$, and $\tilde{\lambda}_{j}$ invertible. Then (for either multiple or distinct roots $\left.\lambda_{j}(a)\right)$

$$
\int \psi^{2}\|P v\|^{2} d t \geqq c^{m} \sum_{i=0}^{m}\left(\begin{array}{c}
m \\
m-l
\end{array}\right) k^{m-2 l} \int \psi^{2}\left\|\Lambda^{l} v\right\|^{2} d t
$$

whenever $t \rightarrow \Lambda^{m-i} v^{(i)} \in \mathscr{E}^{0}(H)$ and $v^{(i)}(0)=v^{(i)}(\tau)=0$ for $0 \leqq i \leqq m-1$. 
Proof. The inequality (4.6) is obtained by $m$ iterations of the procedure indicated in (4.5).

QED

Suppose now that $u$ is a solution of $P u=0$ with $u^{(i)}(0)=0(0 \leqq i \leqq m-1)$. Let $\phi \in C^{m}[0, \infty)$ with $\phi=0$ for $t \geqq \tau$ and $\phi=1$ for $0 \leqq t \leqq \tau / 2$. Set $\phi u=v$ and then $v$ satisfies the requirements of Proposition 5. Also there results

$$
P v=P(\phi u)=\phi P(u)+\Phi=\Phi,
$$

where the support of $\Phi$ lies in $[\tau / 2, \tau]$. Hence

$$
\int_{0}^{\tau} \psi^{2}\|P v\|^{2} d t \leqq \int_{\tau / 2}^{\tau} \psi^{2}\|\Phi\|^{2} d t \leqq c \int_{\tau / 2}^{\tau} \psi^{2} d t
$$

where $c$ depends only on $\phi$ and $u$. As before we may conclude

Theorem 3. Assume the hypotheses of Proposition 5 and that $P u=0$ with $u^{(i)}(0)=0$ for $0 \leqq i \leqq m-1$. Then $u \equiv 0$ in a neighborhood of $t=0$.

REMARK 4. The above result in uniqueness holds even if only a bound $\int \psi^{2}\|P v\|^{2} d t \leqq c \Sigma_{0}^{p} \int \psi^{2}\left\|\Lambda^{l} v\right\|^{2} d t+c \int_{\tau / 2}^{\tau} \psi^{2} d t$ is somehow obtained where $p$ is the largest integer $l$ such that $m-2 l>0$. For example if

$$
\|P u\|^{2} \leqq c \sum_{0}^{p}\left\|\Lambda^{l} u\right\|^{2}
$$

this situation will occur since then

$$
\|\phi P u+\Phi\|^{2} \leqq 2\left(\phi^{2}\|P u\|^{2}+\|\Phi\|^{2}\right) \leqq 2\|\Phi\|^{2}+2 c \Sigma\left\|\Lambda^{l} v\right\|^{2} .
$$

If now $\tilde{\lambda}_{j}=0$ then as in (3.34) we have an estimate $I_{j} \geqq \tilde{c} k \int\|\omega\|^{2} d t$. Similarly if $\tilde{\lambda}_{j}$ is invertible a simpler estimate for $I_{j}$ is given by $I_{j} \geqq(k / 6) \int\|\omega\|^{2} d t$ (see (3.30)). Hence if the $\tilde{\lambda}_{j}$ are either zero or invertible we obtain by iteration

$$
\int \psi^{2}\|P v\|^{2} d t \geqq c^{m} k^{m} \int \psi^{2}\|v\|^{2} d t .
$$

This implies, arguing as before,

THEOREM 4. Assume $D(\Lambda) \subset D\left(\Lambda^{*}\right), \Lambda^{*}-\Lambda$ bounded, and $\lambda_{j}(z)$ analytic on $U^{\varepsilon}$; if $\tilde{\lambda}_{j} \neq 0$ for some $j$ assume $\tilde{\lambda}_{j}$ is invertible and suppose $\left\|\left(a_{i}^{*}-a_{i}\right) \Lambda^{*}\right\| \leqq c\left\|a_{i}\right\|$. Then if $P u=0$ with $u^{(i)}(0)=0$ for $0 \leqq i \leqq m-1$ it follows that $u \equiv 0$ in a neighborhood of $t=0$.

5. We will discuss the case $m=2$ briefly in this section and give some examples. When $m=2, q(\lambda, z)=\lambda^{2}-z_{1} \lambda+q_{0}\left(z_{0}\right)$ with $q_{0}\left(z_{0}\right)=q_{0}+q_{1} z_{0}+q_{2} z_{0}^{2}$. Thus a complete expression for $\lambda_{i}$ is

$$
\lambda=\frac{z_{1}}{2} \pm \frac{1}{2} \sqrt{ }\left(z_{1}^{2}-4 q_{0}-4 q_{1} z_{0}-4 q_{2} z_{0}^{2}\right) .
$$


The discriminant $D(z)=z_{1}^{2}-4 q_{0}-4 q_{1} z_{0}-4 q_{2} z_{0}^{2}$ vanishes in general on a somewhat nontrivial set in $\mathbb{C}^{2}$ even in this simplest of cases. When $\Lambda$ is selfadjoint positive the spectrum of $\Sigma$ lies in the region $z_{0}$ real, $0 \leqq z_{0} \leqq\|\Sigma\|$, and contains zero. Now $\sigma=\sigma\left(\tilde{A}_{1}, \Sigma ; \mathscr{B}\right)$ is the set of points $\left\{\left(\hat{\Sigma}(\phi), \hat{a}_{1}(\phi)\right)\right\}\left(a_{1}=\tilde{A}_{1}\right)$; hence if the spectrum of $\tilde{A}_{1}$ is purely real or imaginary we can plot $\sigma\left(\tilde{A}_{1}, \Sigma ; \mathscr{B}\right)$ in $\mathbb{R}^{2}$. The case represented by $z_{1}$ purely imaginary occurs, e.g., when $\tilde{A}_{1}+\tilde{A}_{1}{ }^{*}$ $=0$; in this case somewhat stronger uniqueness results can be obtained by the methods of [16] (see in particular p. 151). We remark however that no general result seems to have been obtained by such methods for uniqueness without a boundedness condition on the Hermitian part of $A_{1}$ (this would correspond here to a condition of the form $\left(\tilde{A}_{1}+\tilde{A}_{1}^{*}\right) \Lambda$ bounded). Therefore we will consider here the case $z_{1}$ real and show that uniqueness results can be obtained by the methods of this paper. It is seen thereby that although the use of characteristic roots (at least of the kind considered here), and thus a distinction of "type," seems to impose definite limitations on the theory, still it appears to give new results in some cases. A study of this matter is in progress.

For simplicity we assume $q_{1}=0$ and set $p=q_{1}^{2}-4 q_{0} q_{2}=-4 q_{0} q_{2}$ ( $p$ plays a role in classification in the general case). The discriminant is now $D(z)=z_{1}^{2}+p / q_{2}-4 q_{2} z_{0}^{2}=z_{1}^{2}-4 q_{0}-4 q_{2} z_{0}^{2}$ and curves $D(z)=0$ are easily plotted. These show what kind of norm conditions on $\Sigma$ and $\tilde{A}_{1}$ will provide well-determined analytic $\lambda_{i}(z)$ on $\sigma$ as well as distinct $\lambda_{i}$ in the cases considered.

We assume $\sigma$ lies within a region where the radical in (5.1) can be defined as a single-valued analytic function. For $q_{0}>0, D(z)<0$ at the origin and for $q_{0}<0$, $D(z)>0$. Thus when $D(z)>0$ on $\sigma$ from (5.1) we write

$$
\lambda_{i}+\lambda_{i}^{*}=\frac{1}{2}\left(\tilde{A}_{1}+\tilde{A}_{1}^{*}\right) \pm \frac{1}{2}\left[\sqrt{ }\left(\tilde{A}_{1}^{2}-q_{0}(\Sigma)\right)+\sqrt{ }\left(\tilde{A}_{1}^{* 2}-q_{0}(\Sigma)\right)\right]
$$

If $D(z)<0$ on $\sigma$ then a sign change occurs in taking conjugates and we have

$$
\lambda_{i}+\lambda_{i}^{*}=\frac{1}{2}\left(\tilde{A}_{1}+\tilde{A}_{1}^{*}\right) \pm \frac{i}{2}\left[\sqrt{ }\left(q_{0}(\Sigma)-\tilde{A}_{1}^{2}\right)-\sqrt{ }\left(q_{0}(\Sigma)-\tilde{A}_{1}^{* 2}\right)\right]
$$

Thus if $\tilde{A}_{1}=\tilde{A}_{1}{ }^{*}$ for exam ple then in (5.3) $\tilde{\lambda}_{i}=\frac{1}{2} \tilde{A}_{1}$ and $\tilde{\lambda}_{i}$ invertible means $\tilde{A}_{1}$ invertible. In the case of (5.2) and $\tilde{A}_{1}=\tilde{A}_{1}^{*}$ we have $2 \tilde{\lambda}_{i}=\tilde{A}_{1} \pm \sqrt{ }\left(\tilde{A}_{1}^{2}-q_{0}(\Sigma)\right)$; invertibility for this case involves more complicated criteria. The reader is invited to carry out similar calculations when the spectrum of $\tilde{A}_{1}$ is purely imaginary and for the most general cases.

As a final example consider the case $m=2$ with $A_{i}=p_{i}(\Lambda), p_{0}(\Lambda)=a \Lambda^{2}+b \Lambda+c$, $p_{1}(\Lambda)=i d \Lambda+e, p_{2}(\Lambda)=1$. This leads to $q_{2}(\Sigma)=1, q_{1}(\Sigma)=i d+e \Sigma$, $q_{0}(\Sigma)=a+\Sigma b+c \Sigma^{2}$. Then $q(\lambda)$ is given by

$$
q(\lambda)=\lambda^{2}-\lambda(i d+e \Sigma)+\left(a+b \Sigma+c \Sigma^{2}\right)
$$


We have written id above to relate this example to situations in different equations, e.g., $\Lambda=-\partial^{2} / \partial x^{2}$. Thus $q(\lambda, z)=\lambda^{2}-\lambda(i d+e z)+\left(a+b z+c z^{2}\right)$ and the discriminant is $D(z)=-\left(d^{2}+4 a\right)+z(2 i d e-4 b)+z^{2}\left(e^{2}-4 c\right)$ with $\lambda_{i}(z)$ given by $\lambda_{i}(z)=\frac{1}{2}(i d+e z) \pm \frac{1}{2} \sqrt{ } D(z)$. If $a$ is real and $d$ is not pure imaginary then $D(z) \neq \equiv$ and $D(0) \neq 0$; hence if $\|\Sigma\|$ is small enough the roots of $D(z)$ lie outside some $U^{\ell}$. Some criteria in this direction can be found in [18]. It is in. teresting to plot again some diagrams indicating the position of the zeros of $D(z)$ as the constants $a, b, c, d, e$ vary.

\section{REFERENCES}

1. P. Appell et E. Goursat, Théorie des fonctions algébriques, Gauthier-Villars, Paris, 1929.

2. R. Arens, The analytic-functional calculus in commutative topological algebras, Pacific J. Math. 11 (1961), 405-429.

3. - The analytic-functional calculus in commutative Banach algebras, Proc. Sympos. on Linear Spaces, Jerusalem, 1960.

4. R. Arens and A. Calderón, Analytic functions of several Banach algebra elements, Ann. of Math. (2) 62 (1955), 204-216.

5. A. Balakrishnan, Fractional powers of closed operators and the semigroups generated by them, Pacific J. Math. 10 (1960), 419-437.

6. S. Bochner and W. Martin, Several complex variables, Princeton Univ. Press, Princeton,

N. J., 1948.

7. F. Browder, Functional analysis and partial differential equations, Math. Ann. 138 (1959), 55-79.

8. A. Calderón, Uniqueness in the Cauchy problem for partial differential equations, Amer. J. Math. 80 (1958), 16-36.

9. - Integrales singulares y sus aplicaciones a ecuaciones diferenciales hiperbolicas, Seminario, Buenos Aires, 1960.

10. R. Carroll, Some degenerate Cauchy problems with operator coefficients, Pacific J. Math. 13 (1963), 471-485.

11. — Problems in linked operators, I, Math. Ann. 15 (1963), 272-282.

12. - On the singular Cauchy problem, J. Math. Mech. 12 (1963), 69-102.

12a. - Sur la definition spectrale d'un opérateur de Green, C. R. Acad. Sci. Paris 257 (1963), 1663-1665.

13. J. Dieudonné, Foundations of modern analysis, Academic Press, New York, 1960.

14. T. Kato, Integration of the equation of evolution in a Banach space, J. Math. Soc. Japan 5 (1953), 208-304.

15. I. Katz, On the existence of weak solutions to linear partial differential equations, J. Math. Anal. Appl. 2 (1961), 111-144.

16. J. Lions, Équations différentielles-opérationnelles et problèmes aux limites, Springer, Berlin, 1961.

17. B. Malgrange, La méthode de Calderón, Séminaire Schwartz, 1959/60; Unicité du problème de Cauchy, d'après A. P. Calderón, Séminaire Bourbaki 11 (1958/59), No. 178.

18. M. Marden, The geometry of the zerns of a polynomial in a complex variable, Math. Surveys No. 3, Amer. Math. Soc., Providence. R. I., 1949.

19. S. Mizohata, Unicité dans le problème de Cauchy pour quelques équations différentielles elliptiques, Mem. Coll. Sci. Univ. Kyoto 31 (1958), 121-128.

20. J. Neuwirth and R. Carroll, Quelques théorèmes d'unicité pour des équations différentielles-opérationnelles, C. R. Acad. Sci. Paris 255 (1962), 2885-2887. 
21 R. Phillips, Dissipative operators and hyperbolic systems of partial differential equations, Trans. Amer. Math. Soc. 90 (1959), 193-254.

22. C. Rickart, Banach algebras, Van Nostrand, New York, 1960.

23. R. Riesz and B. Sz.-Nagy, Leçons d'analyse fonctionnelle, 2ième éd., Akadémiai Kiadó, Budapest, 1953.

24. G. Šilov, On the decomposition of a commutative normed ring into the direct sum of ideals, Mat. Sb. (N. S.) 32 (74) (1953), 353-364.

25. H. Tanabe, Evolutional equations of parabolic type, Proc. Japan Acad. 37 (1961), 610-613.

26. F. Trèves, Problemes de Cauchy et problèmes mixtes en théorie des distributions, J. Analyse Math. 7 (1959), 105-187.

27. B. van der Waerden, Modern algebra, Ungar, New York, 1947.

28. M. Višik, The Cauchy problem for equations with operator coefficients, the mixed boundary value problem for systems of differential equations, and an approximation method for their solution, Mat. Sb. (N. S.) 39 (1956), 51-148.

29. M. Višik and O. Ladyženskaja, Boundary value problems for partial differential equations and certain classes of operator equations, Uspehi Mat. Nauk 11 (1956), 41-97.

30. L. Waelbroeck, Le calcul symbolique dans les algèbres commutatives, J. Math. Pures Appl. 33 (1954), 147-186.

31. K. Yosida, An operator theoretic integration of the wave equation, J. Math. Soc. Japan 8 (1956), 79-92.

Rutgers, The State University,

NeW BRunswick, NeW JeRSEY 